\title{
Parton Distributions in the Higgs Boson Era
}

\author{
Juan Rojo ${ }^{* \dagger}$ \\ PH Department, TH Unit, CERN, CH-1211 Geneva 23, Switzerland \\ E-mail: juan.rojo@cern.ch
}

\begin{abstract}
Parton distributions are an essential ingredient of the LHC program. PDFs are relevant for precision Standard Model measurements, for Higgs boson characterization as well as for New Physics searches. In this contribution I review recent progress in the determination of the parton distributions of the proton during the last year. Important developments include the impact of new LHC measurements to pin down poorly known PDFs, studies of theoretical uncertainties, higher order calculations for processes relevant for PDF determinations, PDF benchmarking exercises with LHC data, as well as methodological and statistical improvements in the global analysis framework. I conclude with some speculative considerations about future directions in PDF determinations from the theory point of view.
\end{abstract}

XXI International Workshop on Deep-Inelastic Scattering and Related Subjects -DIS2013,

22-26 April 2013

Marseille, France

\footnotetext{
* Speaker.

$\dagger$ This work is supported by a Marie Curie Intra-European Fellowship of the European Community’s 7th Framework Programme under contract number PIEF-GA-2010-272515.
} 
Parton distributions and LHC phenomenology. Precision physics at the LHC requires ever better estimates of PDF uncertainties [1]. The relevance of PDFs for the LHC physics program can be well illustrated by three representative examples. First of all, PDF uncertainties in Higgs production are a fundamental limit to the extraction the Higgs boson couplings from experimental data, and thus degrade the prospects for Higgs characterization [2, 3]. The limiting factor is provided by the spread of the predictions from recent NNLO PDF sets for Higgs production in gluon fusion, shown in Fig. 1. Second, PDFs at large momentum fractions $x$ suffer from substantial uncertainties, due to the lack of experimental constraints. These uncertainties translate into a lack of predictive power for the production of very massive particles in the $\mathrm{TeV}$ range, expected in most New Physics scenarios, such as for heavy supersymmetric particles [4, 5], see also Fig. 1. The final example is provided by the determination of the $W$ boson mass at the Tevatron and at the LHC: this precision observable provides stringent consistency tests of the Standard Model through the global electroweak fit [6], but is currently limited by PDF uncertainties at the Tevatron [7], and will be even more the case at the LHC [8].
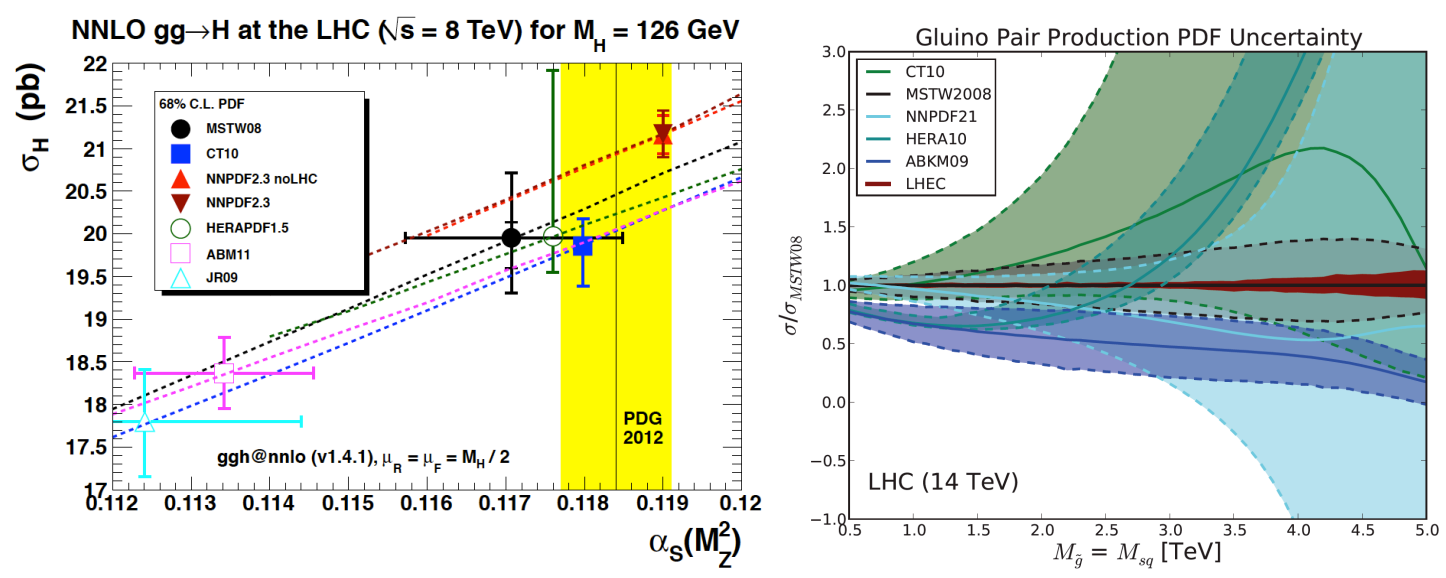

Figure 1: (left) PDF dependence of the Higgs production cross section in gluon fusion at $8 \mathrm{TeV}$ [1]. (right) PDF uncertainties for the production of heavy supersymmetric particles at the LHC, from [4].

The aim of this contribution is to present a succinct overview of recent progress in PDF determinations since DIS2012. More comprehensive recent reviews of PDF developments can be found in Refs. $[1,9,10]$.

PDF updates and benchmarking with LHC data. Various collaborations provide regular updates of their PDF sets. The latest releases from each group are ABM11 [11], CT10 [12], HERAPDF1.5 [13, 14], MSTW08 [15] and NNPDF2.3 [16]. All of these sets provide their PDFs both at NLO and NNLO, and a wide range of values of the strong coupling $\alpha_{s}\left(M_{Z}\right)$ is available. A recent addition to the family of PDF anaysis is the CTEQ-JLAB collaboration with their new CJ12 set [17], which emphasizes the use of large- $x$ DIS data to reduce the experimental PDF uncertainties and an accurate treatment of higher twists and deuteron corrections.

A recent benchmarking exercise comparing NNLO PDFs between them and with Tevatron and LHC data was presented in [18], updating previous benchmarking studies [3]. The comparisons were performed at the level of PDFs, parton luminosities, LHC $8 \mathrm{TeV}$ total cross sections and differential distributions for jet and $W, Z$ data from the Tevatron and LHC $7 \mathrm{TeV}$. A detailed 
discussion was presented concerning the quantification of data versus theory agreement though the use of various $\chi^{2}$ estimators. The main outcome of the comparisons was the confirmation of the reasonable agreement between CT, MSTW and NNPDF, already observed in previous benchmarks. The agreement improves if 2010 NLO PDFs are compared to 2012 NNLO PDFs for a variety of important observables, with the unfortunate exception of gluon-gluon Higgs production for $m_{H}=$ $125 \mathrm{GeV}$. The HERAPDF set leads to similar central values as the global sets but larger PDF uncertainties due to the reduced dataset, while ABM leads to softer large- $x$ gluons and harder quarks as compared to the three global sets. As an illustration, the comparison of the gluon-gluon luminosity between the five NNLO PDF sets considered is shown in Fig. 2. ${ }^{1}$
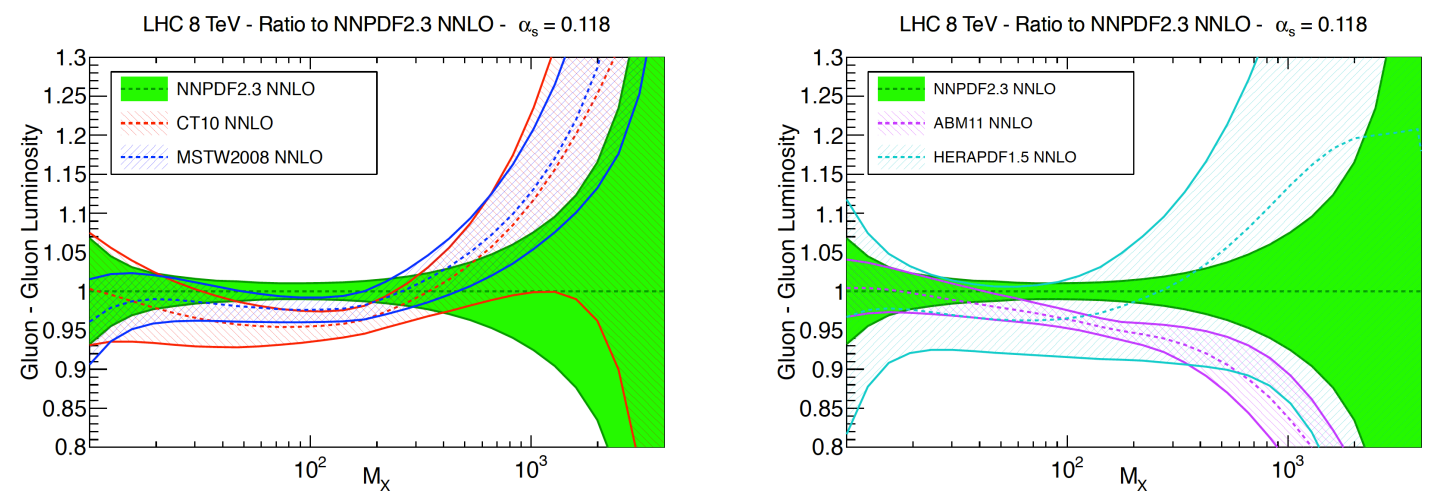

Figure 2: Comparison of the gluon-gluon luminosities as a function of the final state mass $M_{X}$ for the five NNLO PDF sets of the benchmark comparison from Ref. [18].

Constraints from LHC data. A major update since DIS2012 has been the inclusion of LHC data into PDF analysis. Currently, NNPDF2.3 is the only publicly available PDF set that includes constrains from ATLAS, CMS and LHCb jet and $W, Z$ data, but other groups have presented preliminary updates of their fits with the presence of LHC data. For instance, ABM has studied the impact of LHC electroweak boson data into their PDFs in [19, 20].

The traditional processes at hadron colliders used for PDF constraints are inclusive jets and $W, Z$ production. Inclusive jet and dijet data are now available up to the $\mathrm{TeV}$ region from ATLAS and CMS [21, 22], and provide important constrains on the poorly known large- $x$ quarks and gluons. On the other hand, the neutral current Drell-Yan processes has now been measured both in the $Z$ peak region as well as for high and low masses by ATLAS, CMS and LHCb, providing useful information on the large- $x$ quarks and antiquarks (for high mass) and in the small- $x$ gluon and possible departures from lineal DGLAP evolution (for low mass DY).

In addition, thanks to the wealth of LHC data several new processes have become available for their use in PDF fits. The recent calculation of the full NNLO top quark production [23] cross section makes possible for the first time to include top quark data into a NNLO analysis to constrain the large- $x$ gluon PDF [24] (see Fig. 3). Top production is currently the only hadronic observable which is both directly sensitive to the gluon and can be included in a NNLO global fit without

\footnotetext{
${ }^{1}$ An complete extensive set of comparison plots can be found online at the NNPDF HepForge website, https://nnpdf.hepforge.org/html/pdfbench/catalog/
} 
any approximation. In turn, the more accurate gluon PDF translates into an improvement of the theory predictions for various high-mass BSM processes driven by the gluon luminosity. Another interesting result of Ref. [24] is that the gluon determined from a NNPDF2.1 fit to inclusive DIS HERA data only $[25,26]$ and supplemented by LHC top production data is quite close to that of the global fit, driven by jet data, a remarkable consistency test of the global QCD analysis framework. The use of LHC isolated photon data and photon+jet data has also been advocated in order to pin down the gluon PDF [27, 28], though this process is affect by missing higher order and non perturbative uncertainties.
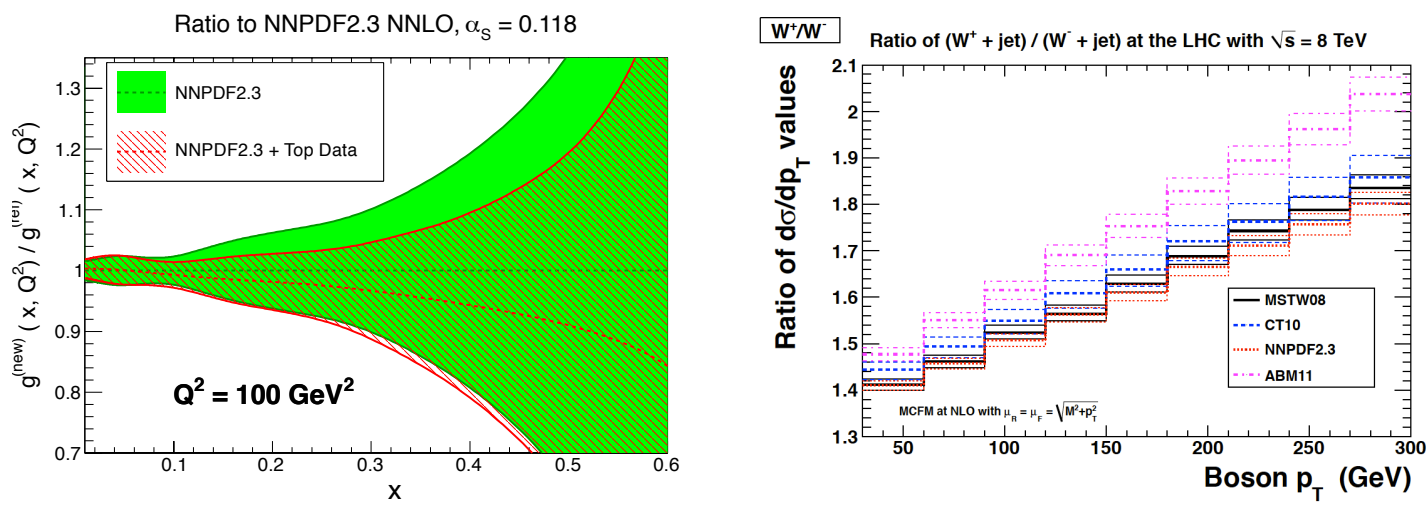

Figure 3: (left) The NNPDF2.3 NNLO gluon at large- $x$ after the inclusion of LHC top quark cross-section data [24]. (right) PDF sensitivity of the ratio $W^{+} / W^{-}$at high values of $p_{T}$ [29].

Turning to the constraints on the quark sector, the production of $W$ and $Z$ bosons in association with jets, for high $p_{T}$ values of the electroweak boson, is a clean probe at the LHC of both quark flavor separation and of the gluon PDF [29]. In particular, ratios of $W$ and $Z$ distributions at large $p_{T}$ provide constrains on quarks and antiquarks while benefiting from substantial cancellations of experimental and theory uncertainties, see Fig. 3. Another important source of information is $W$ production with charm quarks, directly sensitive to the strange PDF [30], the worst known of all light quark flavors. This process has been recently measured with CMS [31], showing good agreement with the strangeness suppression of global PDF fits derived from the neutrino DIS charm production data, while ATLAS [32] prefers a symmetric strange sea, similar as previously derived from their inclusive $W$ and $Z$ data. Including all these datasets into the global PDF fits would then be crucial to determine the optimal strange PDF which accounts for all experimental constraints.

A recently proposed useful observable at the LHC that can be used to enhance the sensitivity to PDFs are cross section ratios between different beam energies. As discussed in [33], the advantage of these ratios is that many theoretical systematics cancel, while at the same time the sensitivity to PDFs is maintained. In addition, many experimental uncertainties cancel in a dedicated measurement of a cross section ratio, so the net result is a very clean probe of differences between PDF sets. A first implementation of this idea has been presented by ATLAS, with the measurement the ratio of inclusive jet cross sections between $2.76 \mathrm{TeV}$ and $7 \mathrm{TeV}$ [34]. The measurement of cross section ratios between 14 and $8 \mathrm{TeV}$ will provide further direct constrains on various PDF combinations, as well as being potentially useful to enhance the sensitivity to BSM contributions as compared to absolute cross sections [33]. 
The list of additional LHC processes that can potentially used for PDF studies includes single top, open heavy quark and charmonium production among others. Is clear that within a few years PDFs based only on collider data only might become competitive with global PDF sets.

Theoretical developements. The theory frontier in PDF determinations is NNLO, however up to recently only a small number of hadron collider processes relevant for PDFs were available at this order, essentially electroweak vector boson production [35, 36, 37]. In the last year, substantial progress has been made in this direction: the full NNLO top quark production cross section was computed [23], the NNLO corrections to jet production in the $g g$ channel became available [38], and the Higgs plus one jet was completed [39], which suggests that the NNLO calculation for W or $\mathrm{Z}$ in association jet might become available in the near future.

While the consequences of experimental uncertainties in PDF fits have been studied extensively, less work has been devoted to the implications of theoretical uncertainties. Recently, a number of studies have become available that attempt to quantify the role of various potential sources of theoretical uncertainties in the determination of the parton distributions. To begin with, the impact of different choices of quark flavor number renormalization schemes in fitting DIS data has been explored by NNPDF [40] and by Thorne [41]. These studies consistently indicate that the fixed-flavor number scheme leads to softer large- $x$ gluons and harder quarks as compared to PDFs determined in general-mass variable flavor number schemes (GM-VFN), suggesting that the use of different quark flavor number renormalization schemes (GM-VFN versus FFN) might account for part of the differences between ABM11 and global PDF fits. As an illustration, we show in Fig. 4 the NNPDF2.3 gluon at a typical LHC scale determined in a fit with FFN scheme, as compared to the default fit in the FONLL-C GM-VFN scheme [42]. In addition, these studies tend to indicate that a somewhat poorer fit quality to the large $Q^{2}$ HERA data is obtained in the FFN as compared to VFN, perhaps related to the missing resummations of DGLAP logarithms [43].

The sensitivity of the global PDF fit to the $W^{2}$ cut, higher twists and deuteron corrections has been also studied by NNPDF and MSTW [44]. The main conclusions are that while with standard $W^{2}$ cuts the impact of higher twists is negligible, deuteron corrections lead to moderate modifications of the $d / u$ ratio, but restricted to the range $0.1 \lesssim x \lesssim 0.5$. The CJ12 analysis [17] has also studied the treatment of deuteron corrections and the role of higher-twist effects which are important at small $W^{2}$.

Another area of theoretical active developement are PDFs with QED corrections. Precision electroweak computations at hadron colliders require PDFs with a combined QCD and QED evolution, and in turn this requires the determination of the photon PDF in the proton. Photon-initiated corrections are relevant for the determination of $M_{W}$, for background estimates of high mass $Z^{\prime}$ and $W^{\prime}$ searches and for other electroweak processes such as $W W$ production at large invariant mass. The only available set up to know is MRST2004QED [45], where the initial photon parametrization is derived from a QED radiation inspired model. Both NNPDF and CT have presented preliminary results towards updated PDF sets with combined QCD and QED corrections. In the NNPDF case [46], the photon PDF is based on the standard artificial neural networks parametrization, without any non-perturbative assumption, and fitted to DIS and LHC electroweak gauge boson production data. In constraining the photon PDF, the direct photon production data from the HERA collider can also be useful [47]. 

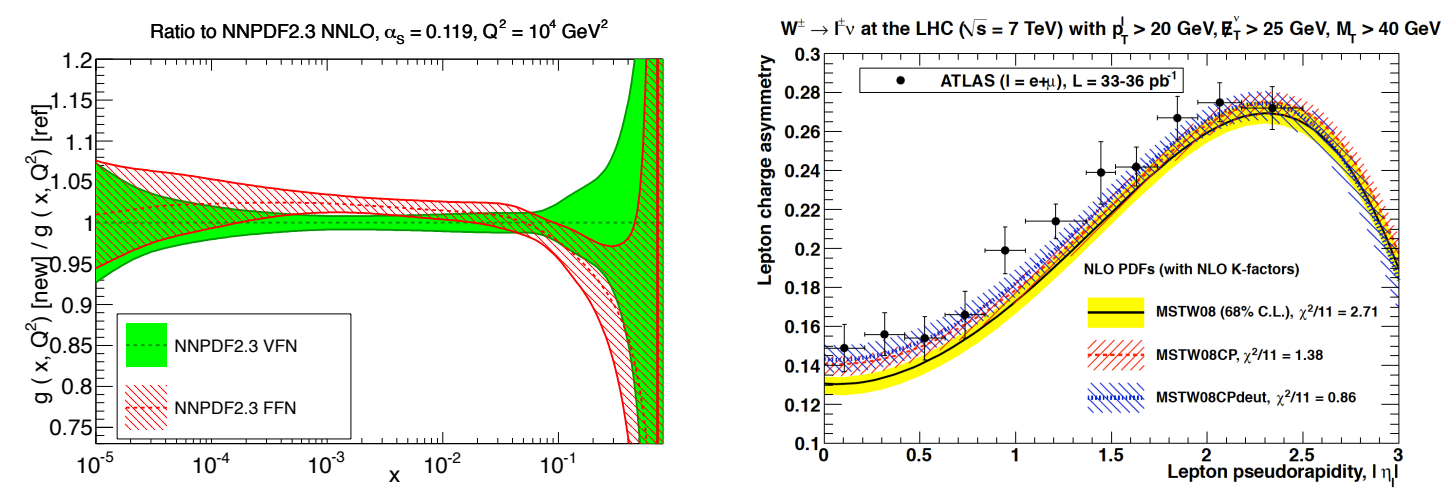

Figure 4: (left) The ratio of gluon PDF at $Q^{2}=10^{4} \mathrm{GeV}^{2}$ extracted using VFN and FFN renormalization schemes in the NNPDF2.3 analysis. (right) The improved description of the LHC $W$ asymmetry data in the MSTW analysis due to the more flexible Chebyshev input PDF parametrization, from [44].

As a last theory issue, the accurate treatment of heavy quark mass effects is important in the fits to HERA cross-section data. The role of heavy quark masses in the description of HERA charm production data has been recently discussed by ABM [48], CT [49] and HERAPDF [50], where the running charm mass $m_{c}\left(m_{c}\right)$ has been extracted from the combined HERA dataset with different treatment of heavy quark structure functions, and the implications for $W$ and $Z$ production at the LHC have been discussed. These results are in general in good agreement with the PDG value.

Methodological studies. Moving to methodology, an important recent development is that of the HERAfitter framework, ${ }^{2}$ an open-source tool for PDF analysis, whose aim is to provide a flexible code, open to contributions from all interested parties, that is able to perform PDF fits, quantify the impact of new data, perform $\chi^{2}$ tests and study the dependence on various theory choices among others. HERAfitter has been used in various experimental analysis by HERA, ATLAS and CMS, and will provide important input both from the theoretical and experimental point of view to global PDF fits. A summary of the HERAfitter project is shown in Fig. 5.

As discussed in the PDF benchmark comparison of Ref. [18], MSTW08 provides a poor description of the $W$ asymmetry data from ATLAS and CMS. This problem can be traced back to a not general enough parametrization of the $u_{V}-d_{V}$ PDF combination. In an update of their global fit, MSTW has presented a more flexible parametrization of their input PDFs based on Chebyshev polynomials [44]. These extended parametrizations lead to an improved description of the LHC $W$ asymmetry data starting from the same non-LHC dataset. This improved description, shown in Fig. 4, is related to a modification in the $u_{V}-d_{V}$ combination that arises when the Chebyshev polynomials are used.

Another important achievement from the methodology point of view is the generalization of the PDF reweighting methods, originally developed for Monte Carlo PDF sets [51, 52], to the case of Hessian based PDFs [53]. These reweighting methods allow to efficiently quantify the impact of new data into PDFs without the need of refitting, for instance, can be used by the experimental collaborations. Therefore, Bayesian PDF reweighting can now be applied to all available PDF sets, both Hessian and Monte Carlo.

\footnotetext{
${ }^{2}$ https: //www.herafitter.org/HERAFitter/
} 

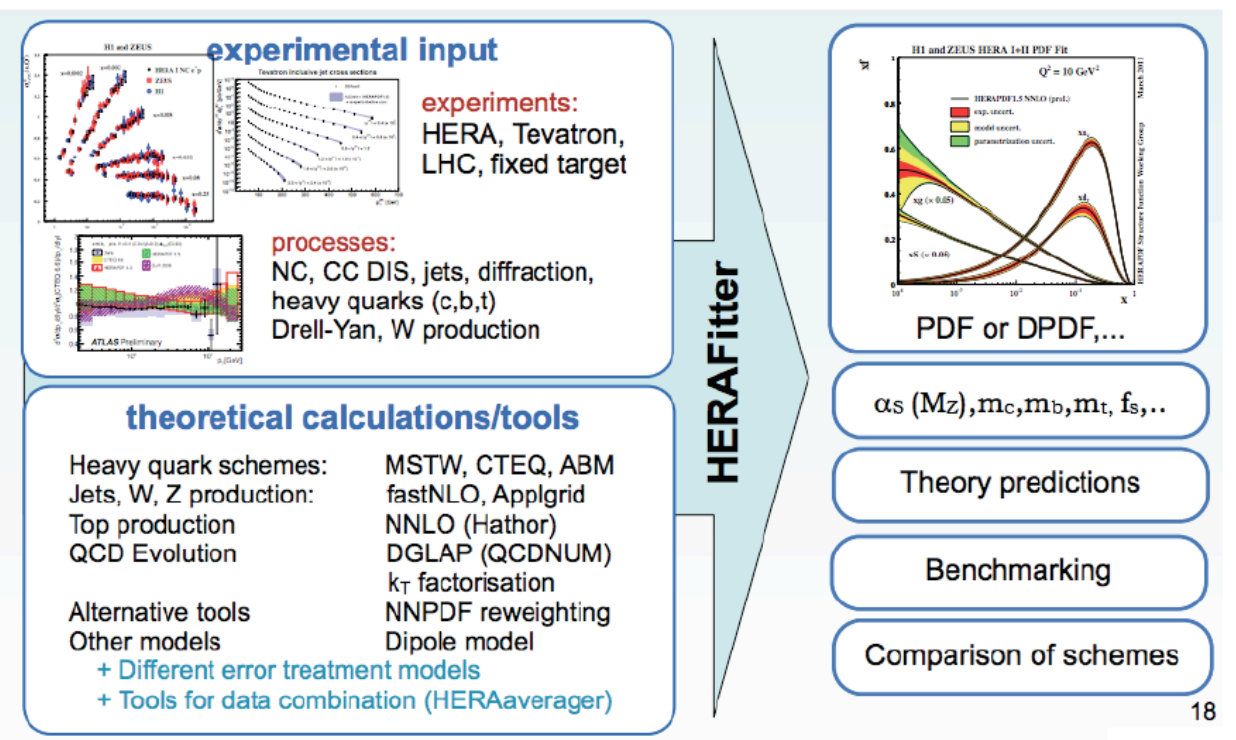

Figure 5: (left) Summary of the HERAfitter project.

One important aspect of the statistical framework of PDF analysis is that various definitions of the figure of merit $\chi^{2}$ exist in the literature, leading to different results when used in the PDF fit. Statistical issues related to these definitions of the $\chi^{2}$ have been discussed recently, see for instance Appendix A of [18]. While different possible treatment of multiplicative uncertainties are possible, only the so-called $T_{0}$ method [54] is strictly unbiased. Note that not only normalization errors are multiplicative, many other experimental systematics, like the jet energy scale, should also be treated as such. In this respect, the CT collaboration has studied the dependence of the gluon PDFs on various possible $\chi^{2}$ definitions of the inclusive jet data [12], showing that at large- $x$ the different $\chi^{2}$ can lead to modifications comparable to the impact of jet data itself. Related issues have also been studied by ABM [55] and earlier by Thorne and Watt [56].

As a last remark concerning methodological improvements, let us mention that in the first NNPDF polarized analysis, NNPDFpol1.0, the determination of the effective large and small- $x$ PDF exponents has been studied [57]. These effective exponents provide useful information to compare the limiting PDF behaviour with non-perturbative models, without the need to introduce an ad-hoc polynomial parametrization.

Theoretical speculations. In the last part of this contribution, let me speculate some possible future directions for possible theory developements in PDF analysis in the coming years:

- Do we need PDFs at (approximate) N3LO, to match the accuracy of foreseen Higgs cross section calculations?

- Do we need PDFs supplemented with threshold resummation, to match the accuracy of processes like Higgs and top production were NNLO+NNLL is the accuracy frontier?

- We need to include electroweak corrections in the fit to the TeV LHC data? Is it enough to include them at the matrix element level or some modification of the DGLAP evolution 
might be needed?

- Do we need specific PDFs for NLO event generators at the LHC? What is the difference between NLO and NLO+PS PDFs? Is it possible to provide a PDF set that provides a simultaneous description of hard scattering and semihard data?

- Are potential intrinsic heavy flavours relevant for LHC phenomenology?

- Can we design new avenues in using the global QCD framework to derive useful constrains on BSM dynamics from LHC data?

Outlook. Driven by the needs of precision LHC physics, there has been substantial progress in PDF determinations in the last year. PDFs are already an important ingredient of the LHC physics program, essential for the characterization of the Higgs boson, and required for the understanding of potential New Physics signals that the LHC hopes to find after the energy upgrade in 2015.

\section{References}

[1] S. Forte and G. Watt, Progress in the Determination of the Partonic Structure of the Proton, arXiv:1301.6754.

[2] S. Dittmaier, S. Dittmaier, C. Mariotti, G. Passarino, R. Tanaka, et al., Handbook of LHC Higgs Cross Sections: 2. Differential Distributions, arXiv:1201.3084.

[3] G. Watt, Parton distribution function dependence of benchmark Standard Model total cross sections at the 7 TeV LHC, JHEP 1109 (2011) 069, [arXiv: 1106.5788 ].

[4] LHeC Study Group Collaboration, J. Abelleira Fernandez et al., A Large Hadron Electron Collider at CERN: Report on the Physics and Design Concepts for Machine and Detector, J.Phys. G39 (2012) 075001, [arXiv:1206.2913].

[5] M. Kramer, A. Kulesza, R. van der Leeuw, M. Mangano, S. Padhi, et al., Supersymmetry production cross sections in pp collisions at $\sqrt{s}=7 \mathrm{TeV}$, arXiv:1206.2892.

[6] M. Baak, M. Goebel, J. Haller, A. Hoecker, D. Kennedy, et al., The Electroweak Fit of the Standard Model after the Discovery of a New Boson at the LHC, Eur.Phys.J. C72 (2012) 2205, [arXiv:1209.2716].

[7] CDF and D0 Collaboration, 2012 Update of the Combination of CDF and DO Results for the Mass of the W Boson, arXiv:1204.0042.

[8] G. Bozzi, J. Rojo, and A. Vicini, The Impact of PDF uncertainties on the measurement of the $W$ boson mass at the Tevatron and the LHC, Phys.Rev. D83 (2011) 113008, [arXiv:1104.2056].

[9] A. De Roeck and R. Thorne, Structure Functions, Prog.Part.Nucl.Phys. 66 (2011) 727-781, [arXiv:1103.0555].

[10] E. Perez and E. Rizvi, The Quark and Gluon Structure of the Proton, Rep.Prog.Phys. 76 (2013) 046201, [arXiv:1208.1178].

[11] S. Alekhin, J. Blumlein, and S. Moch, Parton Distribution Functions and Benchmark Cross Sections at NNLO, Phys.Rev. D86 (2012) 054009, [arXiv: 1202.2281].

[12] J. Gao, M. Guzzi, J. Huston, H.-L. Lai, Z. Li, et al., The CT10 NNLO Global Analysis of QCD, arXiv:1302.6246. 
[13] H1 and ZEUS Collaborations, V. Radescu, Combination and QCD analysis of the HERA inclusive cross sections, PoS ICHEP2010 (2010) 168.

[14] ZEUS and H1 Collaborations, A. Cooper-Sarkar, PDF Fits at HERA, PoS EPS-HEP2011 (2011) 320, [arXiv:1112.2107].

[15] A. D. Martin, W. J. Stirling, R. S. Thorne, and G. Watt, Parton distributions for the LHC, Eur. Phys. J. C63 (2009) 189-285, [arXiv:0901.0002].

[16] R. D. Ball, V. Bertone, S. Carrazza, C. S. Deans, L. Del Debbio, et al., Parton distributions with LHC data, Nucl.Phys. B867 (2013) 244-289, [arXiv:1207.1303].

[17] J. Owens, A. Accardi, and W. Melnitchouk, Global parton distributions with nuclear and finite- $Q^{2}$ corrections, arXiv:1212.1702.

[18] R. D. Ball, S. Carrazza, L. Del Debbio, S. Forte, J. Gao, et al., Parton Distribution Benchmarking with LHC Data, JHEP 1304 (2013) 125, [arXiv: 1211.5142$].$

[19] S. Alekhin, J. Bl§mlein, and S. Moch, Parton Distributions and $\alpha_{s}$ for the LHC, arXiv:1303.1073.

[20] S. Alekhin, J. Bluemlein, and S. O. Moch, ABM11 PDFs and the cross section benchmarks in NNLO, PoS LL2012 (2012) 016, [arXiv: 1302 . 1516].

[21] ATLAS Collaboration, G. Aad et al., Measurement of inclusive jet and dijet production in pp collisions at sqrt(s) = 7 TeV using the ATLAS detector, Phys. Rev. D86 (2012) 014022, [arXiv:1112.6297].

[22] CMS Collaboration, S. Chatrchyan et al., Measurements of differential jet cross sections in proton-proton collisions at $\sqrt{s}=7 \mathrm{TeV}$ with the CMS detector, arXiv:1212.6660.

[23] M. Czakon, P. Fiedler, and A. Mitov, The total top quark pair production cross-section at hadron colliders through $O\left(\alpha_{S}^{4}\right)$, arXiv:1303.6254.

[24] M. Czakon, M. L. Mangano, A. Mitov, and J. Rojo, Constraints on the gluon PDF from top quark pair production at hadron colliders, arXiv:1303.7215.

[25] NNPDF Collaboration, R. D. Ball et al., Impact of Heavy Quark Masses on Parton Distributions and LHC Phenomenology, Nucl. Phys. B849 (2011) 296-363, [arXiv:1101.1300].

[26] NNPDF Collaboration, R. D. Ball et al., Unbiased global determination of parton distributions and their uncertainties at NNLO and at LO, Nucl.Phys. $\mathbf{B 8 5 5}$ (2012) 153-221, [arXiv:1107.2652].

[27] D. d'Enterria and J. Rojo, Quantitative constraints on the gluon distribution function in the proton from collider isolated-photon data, Nucl.Phys. B860 (2012) 311-338, [arXiv: 1202.1762].

[28] L. Carminati, G. Costa, D. D’Enterria, I. Koletsou, G. Marchiori, et al., Sensitivity of the LHC isolated-gamma+jet data to the parton distribution functions of the proton, EPL 101 (2013) 61002, [arXiv:1212.5511].

[29] S. A. Malik and G. Watt, Ratios of W and Z cross sections at large boson $p_{T}$ as a constraint on PDFs and background to new physics, arXiv:1304.2424.

[30] W. Stirling and E. Vryonidou, Charm production in association with an electroweak gauge boson at the LHC, Phys.Rev.Lett. 109 (2012) 082002, [arXiv:1203.6781].

[31] CMS Collaboration, Measurement of associated charm production in $w$ final states at sqrt(s) $=7 \mathrm{TeV}$, CMS-PAS-SMP-12-002. 
[32] ATLAS Collaboration, Measurement of the production of a $W$ boson in association with a charm hadron in pp collisions at sqrt $(s)=7 \mathrm{TeV}$, ATLAS-CONF-2013-045.

[33] M. L. Mangano and J. Rojo, Cross Section Ratios between different CM energies at the LHC: opportunities for precision measurements and BSM sensitivity, JHEP 1208 (2012) 010, [arXiv:1206.3557].

[34] ATLAS Collaboration, G. Aad et al., Measurement of the inclusive jet cross section in pp collisions at $\operatorname{sqrt}(s)=2.76 \mathrm{TeV}$ and comparison to the inclusive jet cross section at $\operatorname{sqrt}(\mathrm{s})=7 \mathrm{TeV}$ using the ATLAS detector, arXiv:1304.4739.

[35] C. Anastasiou, L. J. Dixon, K. Melnikov, and F. Petriello, High precision QCD at hadron colliders: Electroweak gauge boson rapidity distributions at NNLO, Phys. Rev. D69 (2004) 094008, [hep-ph/0312266].

[36] S. Catani, G. Ferrera, and M. Grazzini, W Boson Production at Hadron Colliders: The Lepton Charge Asymmetry in NNLO QCD, JHEP 1005 (2010) 006, [arXiv : 1002 . 3115].

[37] R. Gavin, Y. Li, F. Petriello, and S. Quackenbush, FEWZ 2.0: A code for hadronic Z production at next-to-next-to-leading order, Comput.Phys.Commun. 182 (2011) 2388-2403, [arXiv:1011.3540].

[38] A. G.-D. Ridder, T. Gehrmann, E. Glover, and J. Pires, Second order QCD corrections to jet production at hadron colliders: the all-gluon contribution, arXiv:1301.7310.

[39] R. Boughezal, F. Caola, K. Melnikov, F. Petriello, and M. Schulze, Higgs boson production in association with a jet at next-to-next-to-leading order in perturbative QCD, arXiv:1302.6216.

[40] NNPDF Collaboration, R. D. Ball et al., Theoretical issues in PDF determination and associated uncertainties, arXiv:1303.1189.

[41] R. Thorne, The Effect of Changes of Variable Flavour Number Scheme on PDFs and Predicted Cross Sections, Phys. Rev. D86 (2012) 074017, [arXiv:1201.6180].

[42] S. Forte, E. Laenen, P. Nason, and J. Rojo, Heavy quarks in deep-inelastic scattering, Nucl. Phys. B834 (2010) 116-162, [arXiv: 1001.2312$].$

[43] F. Caola, S. Forte, and J. Rojo, HERA data and DGLAP evolution: Theory and phenomenology, Nucl.Phys. A854 (2011) 32-44, [arXiv: 1007.5405$].$

[44] A. D. Martin, A. J. Mathijseen, W. J. Stirling, R. S. Thorne, B. J. A. Watt, and G. Watt, Extended Parameterisations for MSTW PDFs and their effect on Lepton Charge Asymmetry from W Decays, arXiv:1211.1215.

[45] A. Martin, R. Roberts, W. Stirling, and R. Thorne, Parton distributions incorporating QED contributions, Eur.Phys.J. C39 (2005) 155-161, [hep-ph/ 0411040 ].

[46] S. Carrazza, these proceedings.

[47] ZEUS Collaboration, S. Chekanov et al., Measurement of isolated photon production in deep inelastic ep scattering, Phys.Lett. B687 (2010) 16-25, [arXiv: 0909.4223$].$

[48] S. Alekhin, J. Blumlein, K. Daum, K. Lipka, and S. Moch, Precise charm-quark mass from deep-inelastic scattering, Phys.Lett. B720 (2013) 172-176, [arXiv:1212. 2355].

[49] J. Gao, M. Guzzi, and P. M. Nadolsky, Charm quark mass dependence in a global QCD analysis, arXiv:1304.3494. 
[50] H1 and ZEUS Collaborations, H. Abramowicz et al., Combination and QCD Analysis of Charm Production Cross Section Measurements in Deep-Inelastic ep Scattering at HERA, Eur.Phys.J. C73 (2013) 2311, [arXiv:1211.1182].

[51] R. D. Ball, V. Bertone, F. Cerutti, L. Del Debbio, S. Forte, et al., Reweighting and Unweighting of Parton Distributions and the LHC W lepton asymmetry data, Nucl.Phys. B855 (2012) 608-638, [arXiv:1108.1758].

[52] The NNPDF Collaboration, R. D. Ball et al., Reweighting NNPDFs: the W lepton asymmetry, Nucl. Phys. B849 (2011) 112-143, [arXiv: 1012.0836].

[53] G. Watt and R. Thorne, Study of Monte Carlo approach to experimental uncertainty propagation with MSTW 2008 PDFs, JHEP 1208 (2012) 052, [arXiv: 1205 . 4024].

[54] NNPDF Collaboration, R. D. Ball et al., Fitting Parton Distribution Data with Multiplicative Normalization Uncertainties, JHEP 05 (2010) 075, [arXiv : 0912 . 2276].

[55] S. Alekhin, J. Bluemlein, and S.-O. Moch, Statistical issues in the parton distribution analysis of the Tevatron jet data, arXiv:1211.2642.

[56] R. Thorne and G. Watt, PDF dependence of Higgs cross sections at the Tevatron and LHC: Response to recent criticism, JHEP 1108 (2011) 100, [arXiv: 1106.5789 ].

[57] NNPDF Collaboration, R. D. Ball et al., Unbiased determination of polarized parton distributions and their uncertainties, arXiv:1303.7236. 